\title{
Effect of Ballistic Electrons on the Optical Response of Hyperbolic Metamaterials
}

\author{
Guilherme M. Marega ${ }^{1 *}$, Achiles F. Mota ${ }^{3}$, Ben-Hur V. Borges ${ }^{1}$ and Euclydes Marega Jr. ${ }^{2}$ \\ ${ }^{1}$ São Carlos School of Engineering, University of São Paulo, São Carlos, Brazil \\ ${ }^{2}$ São Carlos Institute of Physics, University of São Paulo, São Carlos, Brazil \\ ${ }^{3}$ Department of Electrical Engineering, University of Brasília, Brasília, Brazil \\ *corresponding author, E-mail: marega.gui@gmail.com
}

\begin{abstract}
This paper presents a theoretical and experimental study of the effect of ballistic electrons on the optical response of MIM (Metal-Insulator-Metal) like hyperbolic metamaterial structures. The simulated model using standard parameters and the experimental optical transmission show a $20 \%$ peak difference due to the presence of ballistic transport in the metal. A semi-analytic approximation based on the Drude's model is used for accurately predicting the optical response of the hyperbolic substrate and plasmon damping in the fabricated metasurfaces.
\end{abstract}

\section{Introduction}

Metamaterials, especially those designed for operating in the visible spectrum, enable deeper control of light. By engineering its primitive components, also called meta-atoms, the light pathway can be precisely controlled. Better and uncommon optical components $[1,2,3]$ can be realized choosing the distribution and the correct meta-atoms.

In special, hyperbolic metamaterials have been attracting attention due to the possibility of light confinement at the nanoscale. This class of metamaterials has its isofrequency surface unbounded and defined by a hyperboloid. While the wave propagation vector $(\vec{k})$ is not restricted to a finite number, the wavelength can achieve infinitly small values $[4,5]$. The only limit for the wavelength compression is defined by the non-ideality physical properties of its constituent material [6] and by the significant losses at highfrequency operation $[7,8]$. This exotic property permits electric field confinement causing a spontaneous emission enhancement leading to a large Purcell factor $[9,10,11]$. Emerging applications on lasing [12], LED's [13, 14] sensing [15, 16], imaging [17, 18] and absorbers [19] have been recently reported showing the interest for continued efforts in this area.

However, in the design of these devices, thin layers of metals with precise thickness are often used. As the metal thickness decreases, the effect of ballistic electrons is neglected as the models are usually fitted for a single thickness. Experimentally, this is seen by the increase of optical attenuation in the transmitted light.

Here, we present a theoretical and experimental study of the effect of ballistic electrons on the optical response of a MIM (Metal-Insulator-Metal) hyperbolic metamaterial structure consisting of silver and silicon dioxide thin films. A semi-analytical model was construct based on the experimental data and the Drude's model with ballistic transport considerations. Finally, the effect of increased electron damping behavior was analyzed relative to the plasmonic behavior of the structure.

\section{Optical response of silver thin films}

Drude's model is the classical way of explaining the optical response of metals. However, when the mean free path of electrons in the material is comparable to its thickness, an extended description must be considered taking into account the effect of ballistic electrons on the material's complex permittivity.

\subsection{Drude Model}

The Drude's model considers that the electrons aren't bounded and move freely in a given metal. Hence, electrons excited by an exterior electromagnetic field move accordingly to the field, only limited by the viscosity of the electrons in the metallic lattice. Mathematically, the movement is similar to a harmonic oscillator, Equation 1, where $m$ : represents the electron mass; $\Gamma$ : the damping factor; $e$ : the electron charge; $\vec{r}(t)$ : the electron movement; and $\vec{E}(t)$ : the electromagnetic excitation.

$$
m \frac{\partial^{2} \vec{r}(t)}{\partial t^{2}}+m \Gamma \frac{\partial \vec{r}(t)}{\partial t}=-e \vec{E}_{0} e^{-i \omega t}
$$

The electronic movement induced by the exterior field defines the optical response of the material. The complex permittivity is given by Equation 2 as a consequence of Equation 1, where $\omega_{p}$ plasma frequency; $\Gamma$ the damping factor; and $\varepsilon_{\infty}$ the offset permittivity due to bounded electrons in the material [20].

$$
\tilde{\varepsilon}(\omega)=\varepsilon_{\infty}-\frac{\omega_{p}^{2}}{\omega^{2}+\Gamma^{2}}+i \frac{\omega_{p}^{2} . \Gamma}{\omega\left(\omega^{2}+\Gamma^{2}\right)}
$$

\subsection{Optical Response of Silver Thin Films}

Physically, the damping has a direct relation to the mean free path of the electron in the metal. The smaller the electron difficult in moving, the longer the mean free path. However, especially for silver which has a mean-free path 
of $53.3 \mathrm{~nm}$ [21], films of the order of tenths of nanometers impose a new boundary on the electron's movement, therefore increasing the damping effect. These electrons have now a ballistic motion due to the interface limitation as the thickness of the film is smaller than the mean-free path.

Equation 3 shows how the interface thickness $(R)$ relates to the damping factor increase $\left(\Gamma^{*}\right)$, where $v_{F}$ : Fermi velocity and $a$ : prefactor dependent on the geometry [20].

$$
\Gamma^{*}=\Gamma+a \frac{v_{F}}{R}
$$

Using equations 2 and 3, we construct the permittivity as function of the wavelength for different thicknesses as shown in Figure 1. The numerical values of silver constants are taken from the data available for silver in Cai and Shalaev [20] where $\Gamma=0.032 \times 10^{15}\left[\mathrm{~s}^{-1}\right]$, $\omega_{p}=14.0 \times 10^{15}[\mathrm{rad} / \mathrm{s}]$ and $v_{F}=1.4 \times 10^{6}[\mathrm{~m} / \mathrm{s}]$ and $a=1$ due to geometric symmetry of a continuous film.

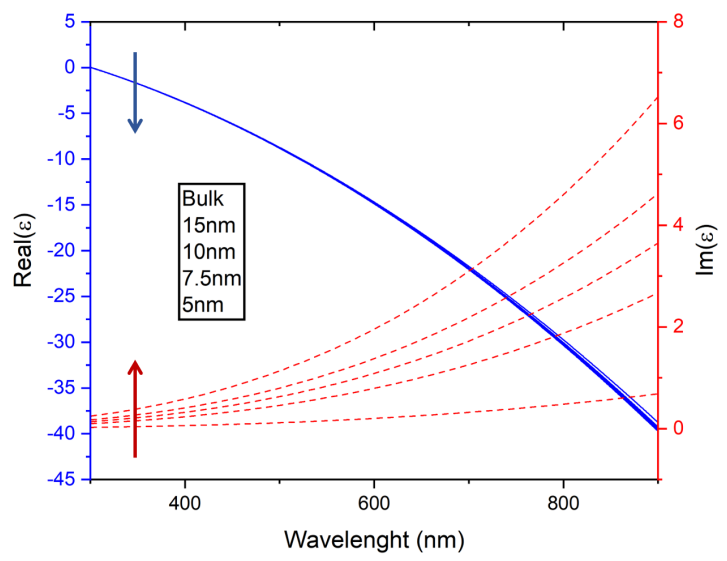

Figure 1: Drude's model of the silver Permittivity as function of the thickness, real part $(\operatorname{Real}(\varepsilon))$ in blue and imagi$\operatorname{nary} \operatorname{part}(\operatorname{Im}(\varepsilon))$ in red.

As shown in figure 1 , the real part of $\varepsilon$ presents a little variation as the thickness changes. Nevertheless, the imaginary part increases as the silver thickness decreases, due to the higher damping factors. Moreover, for larger wavelengths, the difference becomes more prominent.

\subsection{Addition of ballistic effects in Experimental Data}

Although the modeling of the ballistic effect in thin films is expressed analytically in the Drude's model, the real optical behavior of materials takes into account external orbital interactions and atom bounded states. Nevertheless, the available experimental fit for materials usually is done for a single thickness and it does not considers the ballistic effect of electrons. For example, Palik's data for silver consider films 30nm [22] thick. Hence, for verifying the validity of the standard silver model, the transmission of a 30 $\mathrm{nm}$ thermally evaporated silver film is compared to Palik's, CRC's and Jonshon and Christy's models in Figure 2.

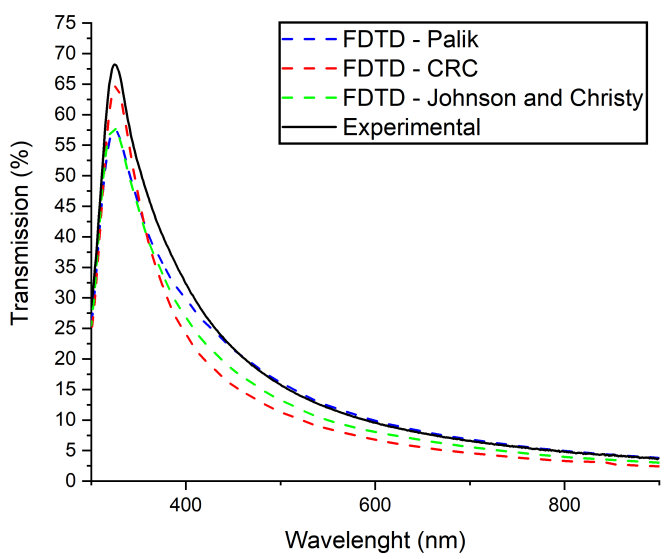

Figure 2: Experimental and simulated transmission of a 30 nm silver film for CRC's, Palik's and Jonhson and Christy's models.

However, the experimental and simulated models are only valid for this thickness due to ballistic electrons effects in thinner thickness. Therefore, we use a semianalytical method for improving the simulation results taking into account ballistic electrons effects. Our approach is capable of calculating the permitivity of thin silver films with any thickness (L) by scalling the experimental data of single thickness using two coeffecients $\alpha_{L(n m)}(\omega)$ and $\beta_{L(n m)}(\omega)$ analytically extracted from the Drude Model. The coefficients $\alpha_{L(n m)}(\omega)$ and $\beta_{L(n m)}(\omega)$ are defined as the ratio between the real and imaginary part, respectively, of the permitivity of a film with thickness L and 30nm obtained via the Drude model as follows,

$$
\begin{aligned}
\alpha_{L(n m)}(\omega) & =\frac{\operatorname{Real}\left(\varepsilon_{\text {Drude }}(\omega)\right)_{L(n m)}}{\operatorname{Real}\left(\varepsilon_{\text {Drude }}(\omega)\right)_{30 n m}} \\
\beta_{L(n m)}(\omega) & =\frac{\operatorname{Im}\left(\varepsilon_{\text {Drude }}(\omega)\right)_{L(n m)}}{\operatorname{Im}\left(\varepsilon_{\text {Drude }}(\omega)\right)_{30 n m}}
\end{aligned}
$$

To calculate the permittivity of a film with thickness $\mathrm{L}$ $(\varepsilon(\omega, L))$ it is necessary to rescale the real and imaginary part of the permittivy of a 30nm thick thilm experimental obtained using the analytical coefficients $\alpha_{L(n m)}(\omega)$ and $\beta_{L(n m)}(\omega)$ as presented Equation (6).

$$
\begin{array}{r}
\varepsilon^{*}(\omega, L)=\alpha_{L(n m)}(\omega) \cdot \operatorname{Real}(\varepsilon(\omega))_{30 n m}+ \\
\quad i \cdot \beta_{L(n m)}(\omega) \cdot \operatorname{Im}(\varepsilon(\omega))_{30 n m}
\end{array}
$$

Figure 3 shows the correction of the experimental data available in the CRC Handbook [23] for silver optical films.

\subsection{Optical Response of Hyperbolic Substrate}

The hyperbolic substrate considered in this paper is a multilayered MIM (Metal-Insulator-Metal) structure (9 in total) over a glass substrate. 


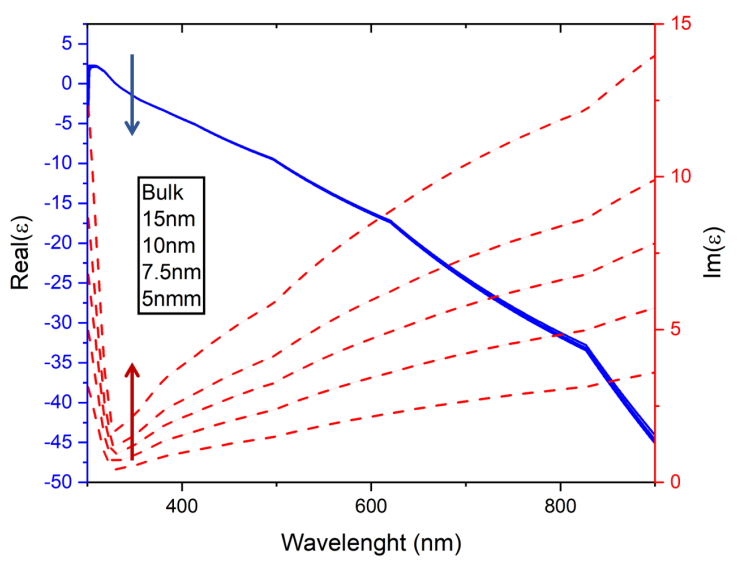

Figure 3: Interpolated silver permittivity experimental data and its correction as function of the wavelength with the thickness as a parameter, Blue(red) curves represent the permittivity's real(imaginary) part.

The top layer is a $7.5 \mathrm{~nm}$ silver layer with the remaining layers consisting of a periodic stack of $15 \mathrm{~nm}$ silver $(\mathrm{Ag})$ and $60 \mathrm{~nm}$ silicon oxide $\left(\mathrm{SiO}_{2}\right)$ [15]. Figure 4(a) is the three dimensional model of such structure.

The fabrication of this film is done by alternating cycles of thermal evaporation for silver layers, and e-beam evaporation for the silicon dioxide layers. The thickness was controlled via standard oscillator frequency shift method embedded in both evaporators, and the total thickness was controlled by a Dektak XT Profilometer.

Figure 4(b) shows the film optical response for experimental and simulated substrates. The experimental optical response is obtained using a UV-Vis spectrometer. The optical transmission of the hyperbolic substrate is numerically obtained with the software FDTD Lumerical ${ }^{\circledR}$ considering initially the standard CRC model, and then, the CRC model with the proposed correction described in the previous section.Figure 4(c) shows the simulated electric fields for different wavelengths.

\subsection{Optical Response of Hyperbolic Metasurfaces}

In this work, two metasurfaces were studied, a linear array of slits with $250 \mathrm{~nm}$ period and $50 \mathrm{~nm}$ width and a concentric array of circular rings with $50 \mathrm{~nm}$ width separated by $350 \mathrm{~nm}$. They were fabricated over a $20 \mu \mathrm{m} \times 20 \mu \mathrm{m}$ surface. Both structures were fabricated using a focused ion beam for milling the pattern on the multilayer structure.

Figures 4(a) and 5(a) show the 3D modeling of the structures, and figures 4(b) and 5(b) the SEM images of the fabricated structure.

The simulation of both structures was carried out using the previous model with ballistic effects using symme-

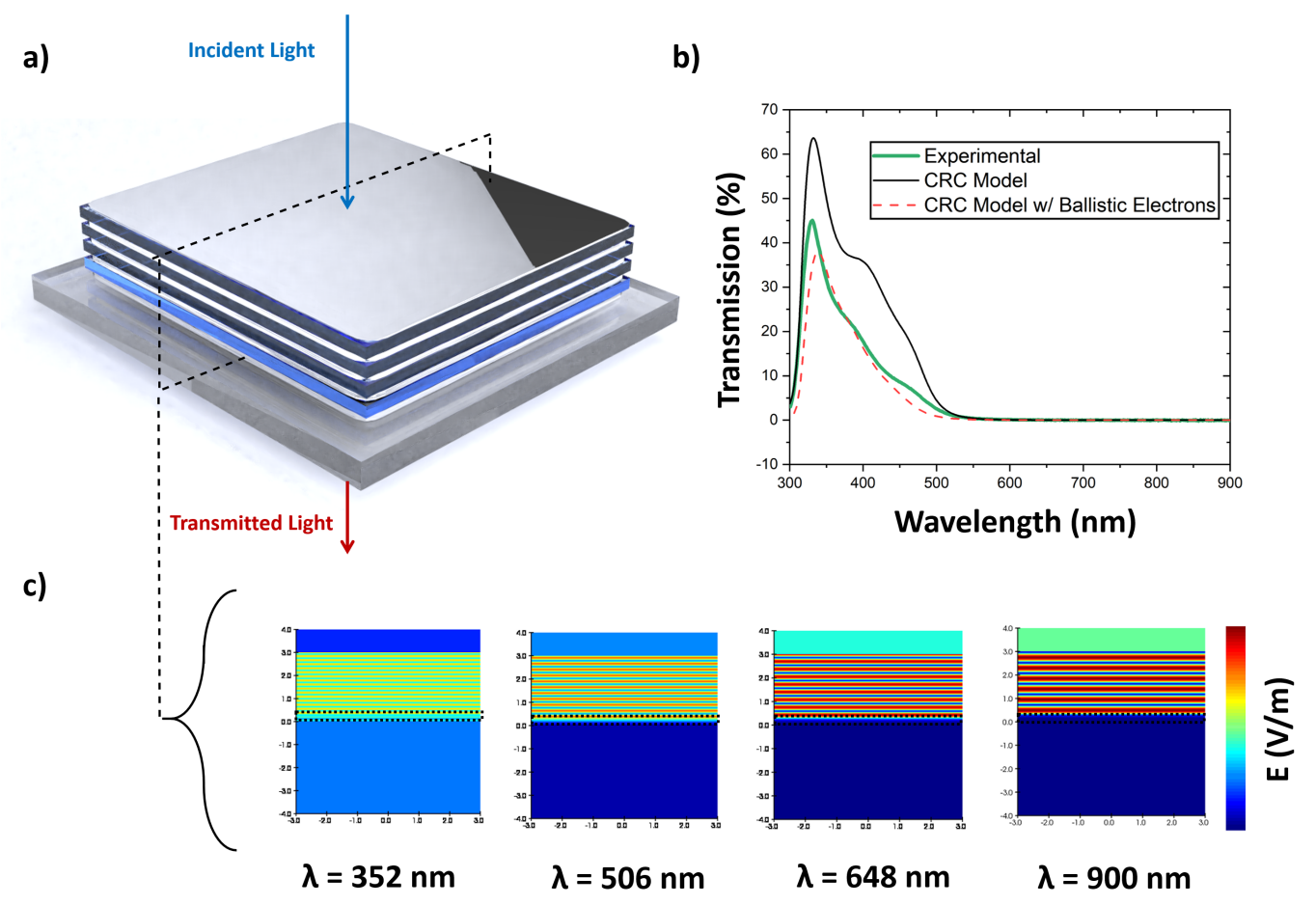

Figure 4: (a) 3D model of the MIM like substrate. (b) Experimental and simulated transmission spectrum considering the model with (dashed red line) and without (solid black line) ballistic electrons effects. (c) Electric field maps in each wavelength. 

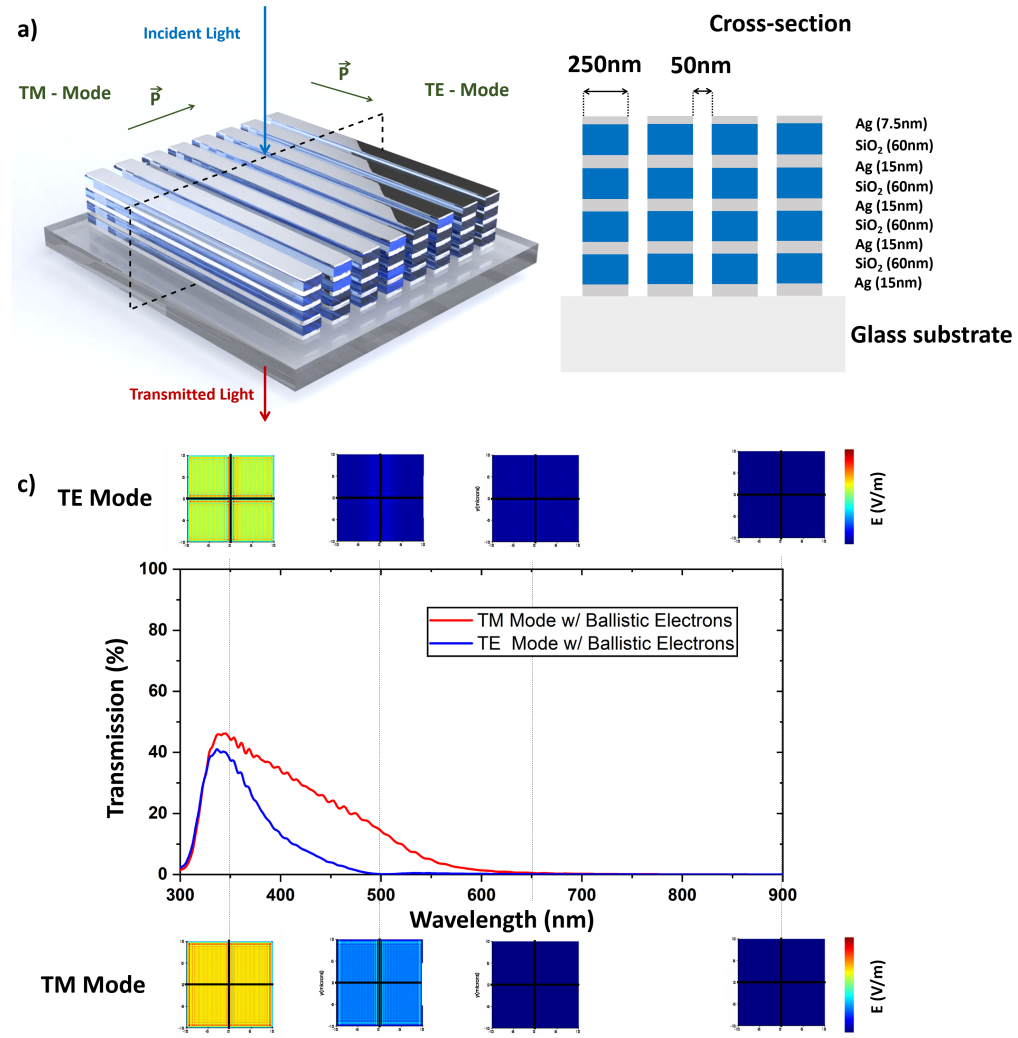

b)

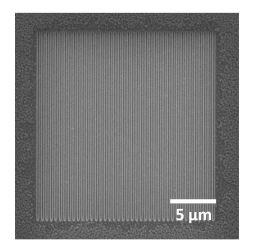

d)

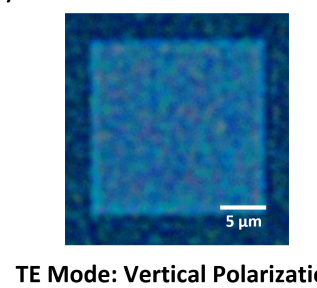

e)

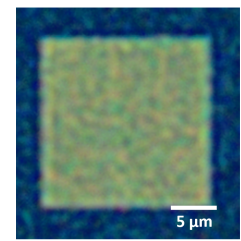

TM Mode: Horizontal Polarization

Figure 4: (a) 3D model of a linear array of slits on a metasurface. (b) SEM image of a linear array of slits with $250 \mathrm{~nm}$ period and $50 \mathrm{~nm}$ width. (c) Simulated normalized transmission spectrum of the metasurface for TE and TM modes and field maps. (d) and (e) are the far-field optical images of the transmitted through the film and the metasurface region as indicated in (b) with the incoming light polarized parallel (TE) to the slits and perpendicular (TM).

tries for reducing memory requirements for mesh size while keeping a precise mesh within the structures. 128 Perfect Matched Layers were used for absorbing outgoing waves. Figures 4(c) and 5(c) show the structure optical transmission for both metasurfaces and the computed electric fields for different wavelengths. For the case of a periodic array of linear slits, two different polarizations were used for exciting the TE (transverse electric, electric field parallel to the slits) and TM (transverse magnetic, electric field perpendicular to the slits), respectively. As for the array of concentric rings just one simulation was carried out due to its invariant with respect to polarization, but considering both standard and corrected models for the silver films.

Figures 4(d-e) present the optical image of the structures obtained by shining white light onto the linear array using an Olympus transmission microscope. Each mode was excited by rotating a linear polarizer placed before the sample.

Finally, Figure 5(d) presents the optical image of the structures obtained by shining white light onto the ring array using a Olympus transmission microscope. Figure 5(e) presents the image from Figure 5(d) using a 594nm highpass filter.

\section{Discussions}

Figure 4(b) shows the simulated results obtained with both models(standard and corrected) and experimental results with almost the same behavior in the blue spectral range. However, as can be seen in Figure 4(b), the transmission calculated with the proposed correction presents a much better agreement with the experimental curve than the CRC model without the correction. The CRC model does not take the ballistic electron into account, which outcomes in a smaller permittivity imaginary part. Hence, in a higher transmission as observed in Figure 4(b), the experimental data shows a peak transmission of about $20 \%$ lower than the one simulated using bulk silver parameters. The mismatch observed near the pick happens due to an interpolation inaccuracy in the simulation results.

This same effect must be considered in the treatment of the light transmitted through the metasurfaces region. As the electron mobility decreases, the plasmon quality may also decrease due to the greater material resistance. Using the semi-analytic approximation, a more reliable simulation of the proposed metastructures in the Figures 4(a) and 5(a) can be obtained.

The optical transmission of the linear array of slits for TE polarization, 4(c), shows a blue spectra similar to the 


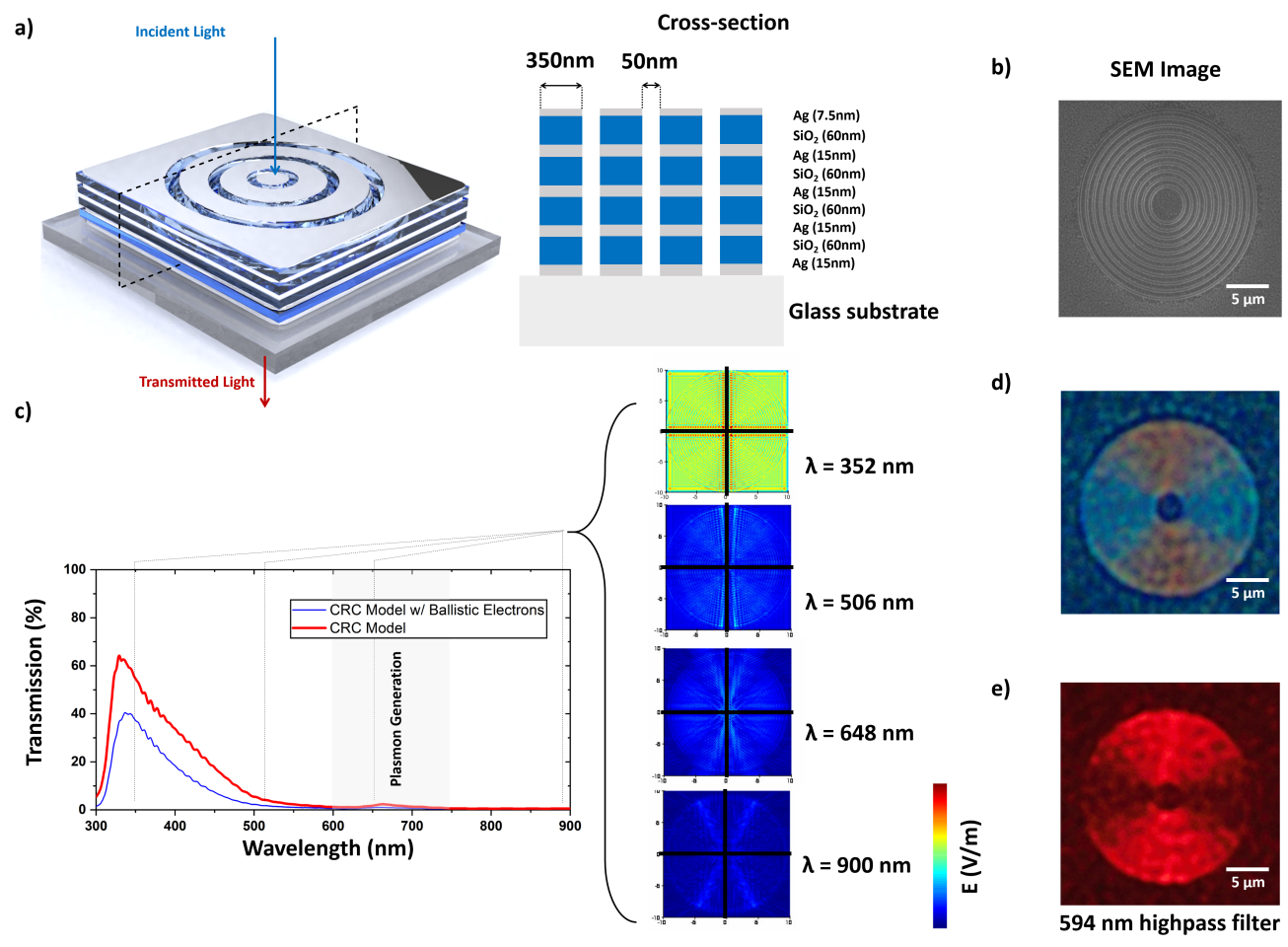

Figure 5: (a) 3D model of the concentric array of circular rings hyperbolic metasurface. (b) SEM image of a concentric array of circular rings with $50 \mathrm{~nm}$ width separated by $400 \mathrm{~nm}$. (c) Simulated normalized transmission spectrum of the metasurface and field maps. (d) far-field optical image of the light transmitted through the metasurface indicated in (b) after a light polarized in the horizontal axis. (e) is the (d) image with 594nm high pass filter has been applied (red filter).

one observed in Figure 4(d). The TM polarized wave, in turn, shows a blue-green spectra similar to the one observed in Figure 4(e). Similarly, the same is observed for the array of concentric rings. The simulation predicts a weak red signal with emission in two principal lobes which are verified in the optical image using a red filter. The blue light transmission seen in the spectrum is due to the transmission mainly from the substrate without interacting with the structure. Also, Figure 5(c) shows the damping in the plasmonic response while considering the ballistic transport.

\section{Conclusions}

This paper has investigated the effect of ballistic electrons in silver thin films and how they impact the attenuation factor of simulated structures when compared to experimental results of hyperbolic metasurfaces. The ballistic transport also affects the plasmon quality in the structure due to increased resistance in its transport. A proposed semianalytical approximation based on ballistic electrons effects in the Drude's model is used to accurately predict the optical response of hyperbolic metasurfaces.

\section{Acknowledgement}

Acknowledgements to The São Paulo Research Foundation (FAPESP) under the grant 2018/10011-3 and 2013/072761. Also, acknowledgements to the Brazilian National Council for Scientific and Technological Development (CNPq) under the grant 310925/2017-7.

\section{References}

[1] PENDRY, John Brian."Negative refraction makes a perfect lens", Physical review letters, v. 85, n. 18, p. 3966, 2000.

[2] J. B. Pendry, D. Schurig, and D. R. Smith, "Controlling Electromagnetic Fields", Science, vol. 312, no. 5781, pp. 17801782, 2006.

[3] W. Cai, U. K. Chettiar, A. V. Kildishev, and V. M. Shalaev, "Optical cloaking with metamaterials", Nature Photonics, vol. 1, no. 4, pp. 224227, Apr. 2007.

[4] P. Huo, S. Zhang, Y. Liang, Y. Lu, and T. Xu, "Hyperbolic Metamaterials and Metasurfaces: Fundamentals and Applications", Advanced Optical Materials, vol. 7, no. 14, p. 1801616, Jul. 2019. 
[5] A. D. Boardman et al., "Waves in hyperbolic and double negative metamaterials including rogues and solitons”, Nanotechnology, vol. 28, no. 44, p. 444001, Oct. 2017.

[6] C. A. Valagiannopoulos and S. A. Tretyakov, "Emulating hyperbolic-media properties with conventional structures", New J. Phys., vol. 16, no. 6, p. 063004, Jun. 2014.

[7] A. Poddubny et al.,Hyperbolic metamaterials, Nature Photonics, vol. 7, no. 12, pp. 948957, Dec. 2013.

[8] Huo, P., Zhang, S., Liang, Y., Lu, Y., Xu, T., Hyperbolic Metamaterials and Metasurfaces: Fundamentals and Applications. Advanced Optical Materials 2019, 7, 1801616.

[9] Jacob, Z., Smolyaninov, I. and Narimanov, E., "Broadband Purcell effect: radiative decay engineering with metamaterials." Applied Physics Letters 100, 181105 (2012).

[10] D. Lu, J. J. Kan, E. E. Fullerton, and Z. Liu, ”Enhancing spontaneous emission rates of molecules using nanopatterned multilayer hyperbolic metamaterials", Nature Nanotechnology, vol. 9, no. 1, pp. 4853, Jan. 2014.

[11] P. Shekhar, J. Atkinson, and Z. Jacob, "Hyperbolic metamaterials: fundamentals and applications", Nano Convergence, vol. 1, no. 1, p. 14, Jun. 2014.

[12] H. I. Lin et al., "Integration of Nanoscale Light Emitters and Hyperbolic Metamaterials: An Efficient Platform for the Enhancement of Random Laser Action." ACS Photonics 5, 718727 (2018). ACS Photon. 5, 718727 (2018).

[13] K. C. Shen et al., Giant enhancement of emission efficiency and light directivity by using hyperbolic metacavity on deep-ultraviolet AlGaN emitter, Nano Energy 45,353358 (2018).

[14] K. L. Tsakmakidis, R. W. Boyd, E. Yablonovitch, and $\mathrm{X}$. Zhang, Large spontaneous-emission enhancements in metallic nanostructures:towards LEDs faster than lasers, Optics Express 24, 1791617927 (2016).

[15] A. F. Mota et al., "2D semi-analytical model for optimizing the radiation emission of quantum emitters embedded in a bounded nano-patterned hyperbolic metamaterial," 2018 12th International Congress on Artificial Materials for Novel Wave Phenomena (Metamaterials), Espoo, 2018, pp. 128-130.

[16] C. L. Degen, F. Reinhard, and P. Cappellaro, Quantum sensing, Review of Modern Physics 89, 139 (2017).

[17] Liu, Z., Lee, H., Xiong, Y., Sun, C. Zhang, X. ”Farfield optical hyperlens magnifying sub-diffractionlimited objects.” Science 315, 1686 (2007).
[18] Ishii, S. et al., "Sub-wavelength interference pattern from volume plasmon polaritons in a hyperbolic medium”. Laser Photonics Review 7, 265271 (2013).

[19] C. A. Valagiannopoulos and I. S. Nefedov, "Increasing the electromagnetic attenuation below a quasimatched surface with use of passive hyperbolic metamaterials", Photonics and Nanostructures - Fundamentals and Applications, vol. 11, no. 3, pp. 182190, Aug. 2013.

[20] W. Cai and V. Shalaev, "Optical Metamaterials". Springer New York, 2010.

[21] GALL, D. "Electron mean free path in elemental metals." Journal of Applied Physics,v. 119, n. 8, p. 085101, 2016.

[22] E.D. Palik, "Handbook of Optical Constants of Solids”, Academic Press, Orlando, 1985

[23] WEBER, M. J., "Handbook of Optical Materials". [S.1.]: CRC Press, 2002. 